\title{
Robust Beamforming for Multibeam Satellite Communication in the Face of Phase Perturbations
}

\author{
Xin Zhang, Jingjing Wang, Student Member, IEEE, Chunxiao Jiang, Senior Member, IEEE, \\ Chaoxing Yan, Yong Ren, Senior Member, IEEE and Lajos Hanzo, Fellow, IEEE
}

\begin{abstract}
A precoding scheme is proposed for the downlink of multibeam satellite communication in the face of phase perturbations. In order to alleviate the signal-to-interference and noise ratio (SINR) degradation induced by phase uncertainty and to minimize the transmission power consumption, we formulated the beamforming problem as a chance-constrained optimization. Moreover, we provided a large deviation inequality (LDI) aided conservative approximation for the chance-constraints, followed by conceiving a tractable solution. Our simulation results showed the accuracy and the efficiency of our proposed approximation algorithm compared to the benchmarks.
\end{abstract}

Index Terms-Multibeam satellite, robust beamforming, phase perturbations, large deviation inequality.

\section{INTRODUCTION}

$\mathbf{T}$ HE design of multicast multibeam satellite communication systems has attracted much attention as a benefit of providing interactive broadband services to remote geographical areas at a high quality of service (QoS) [1]. Multibeam joint beamforming is conducive to full frequency reuse and interference mitigation [2]. In [1], Christopoulos et al. demonstrated that a broadband multibeam satellite communication system relying on a frame-based Transmit PreCoding (TPC) scheme had a higher throughput than the conventional four-color frequency reuse configuration. Meanwhile, Zheng et al. [3] proposed a generic optimization framework of linear precoding design for handling objective functions of data rate, which was close to the dirty paper coding under nonlinear power constraints in general multibeam satellite systems. Furthermore, Joroughi et al. [4] investigated on-board beamforming schemes in a joint multiple gateways architecture and showed that the number of gateways remained affordable even though the data demand increased. Taking both system's

Copyright (c) 2019 IEEE. Personal use of this material is permitted. However, permission to use this material for any other purposes must be obtained from the IEEE by sending a request to pubs-permissions @ieee.org.

X. Zhang, J. Wang and Y. Ren are with the Department of Electronic Engineering, Tsinghua University, Beijing, 100084, China. E-mail: zhangxin15@mails.tsinghua.edu.cn, chinaeephd@gmail.com, reny@tsinghua.edu.cn.

C. Jiang is with Tsinghua Space Center, Tsinghua University, Beijing, 100084, China. E-mail: jchx@ @singhua.edu.cn.

C. Yan is with the Beijing Research Institute of Telemetry, Beijing, China. E-mail: chaoxingyan@foxmail.com.

L. Hanzo is with the School of Electronics and Computer Science, University of Southampton, Southampton, SO17 1BJ, UK. Email: lh@ecs.soton.ac.uk.

L. Hanzo would like to acknowledge the financial support of the EPSRC projects EP/Noo4558/1, EP/PO34284/1, of the Royal Society's GRFC Grant as well as of the European Research Council's Advanced Fellow Grant QuantCom. performance and user's fairness into account [5], Jiang et al. proposed a multimedia multicast beamforming scheme for improving the total system's capacity for the integrated terrestrial-satellite networks.

However, satellite communication systems are characterized by a long round-trip delay (RTD) and imperfect channel state information (CSI) [6]. To elaborate, Vázquez et al. [7] demonstrated that the outdated CSI was expected to degrade the performance of precoded L-band mobile multibeam satellite systems. Naturally, the antenna weights are also affected by the imperfect CSI, which leads to an inaccurate precoding matrix. Hence, it is necessary to consider how the imperfect CSI affects the precoder design. In order to counteract the signal-tointerference and noise ratio (SINR) degradation caused by the CSI estimation error, a robust beamforming has been proposed. There are two types of method invoked for describing the CSI imperfections, namely the so-called determined bounded model [8] and the random model [9]. Specifically, Wang et al. [8] maximized the worst-case received SINR by solving a max-min optimization problem under a bounded uncertainty for multi-input multi-output (MIMO) systems, which may not necessarily result in a better convex restrictive approximation. Another one is constituted by the outage-constrained problem, where the constraints are represented in the form of probability inequalities due to the characteristics of the CSI error, which makes the computation of the antenna weights difficult and hence tackling this challenge is the main focus of our paper. To address this issue, a flexible outage-probability-constrained robust beamforming mechanism is conceived in this paper.

The magnitude of typical satellite channel models, such as line-of-sight (LOS) channel [10] and rain fading channel [11], is determined only by the path attenuation, which usually varies slowly and is easy to estimate [12]. Therefore, we can assume that the amplitude is constant and known during the feedback interval. However, there is a significant variation in the channel phase component arising out of time varying phase perturbations and the high RTD will induce the outdated CSI [11]. To qualify the service of satellite, Li et al proposed a secure and robust beamforming framework for cognitive satellite-terrestrial networks (CSTNs) to minimize the transmit power under a stochastic model for the CSI uncertainty [9]. However, most of the existing contributions dedicated only limited attention to the phase perturbations induced by a number of impairments, such as the presence of the satellite's non-linear transponders and conditioning filters, the channel fading and the imperfect synchronization caused by the oscillator [13]. Moreover, since the phase noise 
increases as the center frequency increases, in particular for the frequency bands beyond Ka-band, the phase perturbation is an important factor in satellite communications. Given these phase perturbations, the probabilistic constraints are reformulated into random complex exponential forms which result in computational challenges. In this paper, we provide a conservative approximation and a tractable solution with the following new contributions:

- Our proposed robust beamforming formulation minimizes the power, while satisfying the SINR constraints in the face of the phase perturbations.

- In order to solve the problem under the probabilistic constraints involving random complex exponential forms relying on the classic Taylor series and large deviation inequality (LDI), we proposed a conservative approximation of the outage probability constraints to give a tractable solution.

- Extensive simulations are conducted in order to show the efficiency of our proposed robust beamformer and to strike a compelling outage probability vs power consumption trade-off.

Notations. Throughout this paper, we use boldfaced lowercase letters to represent column vectors and uppercase Roman letters to represent matrices. $\operatorname{vec}(\mathbf{A})$ represents the vector obtained by joining the column vectors of $\mathbf{A}$. The notations $\mathbb{S}^{N}$ and $\mathbb{K}^{N}$ denote the sets of $N$-order symmetric matrices and skewed-symmetric matrices, respectively. Furthermore, $\operatorname{diag}(\boldsymbol{a})$ represents a square diagonal matrix with the elements of the vector $\boldsymbol{a}$ constituting the main diagonal. The superscripts ' $\mathrm{T}$ ' and ' $\mathrm{H}$ ' represent the transpose and the Hermitian transpose, respectively, while $\operatorname{Tr}(\mathbf{A})$ and $\odot$ represent the trace and the entry-wise product respectively. Additionally, $J_{i}(\cdot)$ stands for the $i$ th-order Bessel functions of the first-kind, while $\|\cdot\|$ and $\|\cdot\|_{F}$ represent the vector Euclidean norm and matrix Frobenius norm respectively. Finally, $\operatorname{Pr}\{\cdot\}$ denotes the probability function.

The remainder of this paper is summarized as follows. In Section $\mathrm{II}$. we describe the system model and formulate the multibeam beamforming problem considering phase perturbations. In Section III, an LDI approximation algorithm is proposed for minimizing the power consumption. In Section IV. the power consumption and the outage probability are used for benchmarking the performance of our proposed model, followed by our conclusions in Section $\mathrm{V}$

\section{Multibeam Beamforming Considering Phase PERTURBATIONS}

In this section, we consider a communication scenario, where terrestrial users can be served by a gateway and a geosynchronous earth orbit (GEO) satellite. To elaborate, the data packets are firstly precoded at the gateway and then sent to the GEO satellite, as shown in Fig. 11. The users receive the data relayed by the GEO satellite.

Although the channel between the gateway and the GEO satellite is not perfect, the digital video broadcasting satellite (DVB-S2) working group [13] assumes that the downlink noise between the satellite and the users dominates the link's overall noise contributions. In line with this, we also assume a noiseless channel between the gateway and the GEO satellite. In our model, the gateway is equipped with $K$ antennas and has realistic imperfect CSI. The GEO satellite also has $K$ antennas, forming a cluster of $K$ adjacent beams directed to the ground. The total bandwidth is $B$. Moreover, time division multiplexing is invoked. Given the strict power constraint of the solar-powered satellite, we formulate the multibeam beamforming as a power minimization problem.

\section{A. Signal Model}

In our model, each signal is precoded at the gateway and transmitted to the GEO satellite. We assume that there are $I$ users and let $s_{i}(t), i=1, \ldots, I$ be the initial signal requested by each user. Then, the transmitted signal $\boldsymbol{x}(t)$ of the GEO satellite precoded by the gateway is given by:

$$
\boldsymbol{x}(t)=\sum_{i=1}^{I} \boldsymbol{w}_{i} s_{i}(t),
$$

where $\boldsymbol{w}_{i}$ represents the precoding vector. Hence, the signal received by the $i$-th user is:

$$
r_{i}(t)=\boldsymbol{h}_{i}^{H} \boldsymbol{w}_{i} s_{i}(t)+\sum_{j \neq i} \boldsymbol{h}_{i}^{H} \boldsymbol{w}_{j} s_{j}(t)+n_{i}(t),
$$

where $n_{i}(t)$ is the additive white Gaussian noise (AWGN) with variance of $\tau_{i}^{2}$. Moreover, $\boldsymbol{h}_{i}=\left(h_{i 1}, \ldots, h_{i K}\right)^{T}$ denotes the channel matrix, where $h_{i j}=\left|h_{i j}\right| e^{j \theta_{i j}}$ is the sub-channel function between the $i$-th user and the $j$-th antenna of GEO satellite. Then, the SINR of the $i$-th user can be formulated as:

$$
\operatorname{SINR}_{i}=\frac{\left|\boldsymbol{h}_{i}^{H} \boldsymbol{w}_{i}\right|^{2}}{\sum_{j \neq i}\left|\boldsymbol{h}_{i}^{H} \boldsymbol{w}_{j}\right|^{2}+\tau_{i}^{2}}
$$

\section{B. Channel Model}

In our model, we have perfect amplitude information of $\left|h_{i j}\right|$, but imperfect phase information, which means that $\hat{\theta}_{i j}=\theta_{i j}-\delta_{i j}$, where $\hat{\theta}_{i j}$ represents the estimate of the phase and $\delta_{i j}$ is its estimation error. Moreover, $\boldsymbol{\Delta}_{i}=\left(\delta_{i 1}, \ldots, \delta_{i K}\right) \sim$ $\mathcal{N}\left(\mathbf{0}, \mathbf{C}_{i}\right)$, where $\mathbf{C}_{i}$ is the correlation matrix. Hence, the estimate of the sub-channel function can be formulated as $\hat{h}_{i j}=\left|h_{i j}\right| e^{j \hat{\theta}_{i j}}$ and we have:

$$
\boldsymbol{h}_{i}=\hat{\boldsymbol{h}}_{i} \odot \boldsymbol{e}_{i}=\operatorname{diag}\left(\hat{\boldsymbol{h}}_{i}\right) \boldsymbol{e}_{i},
$$

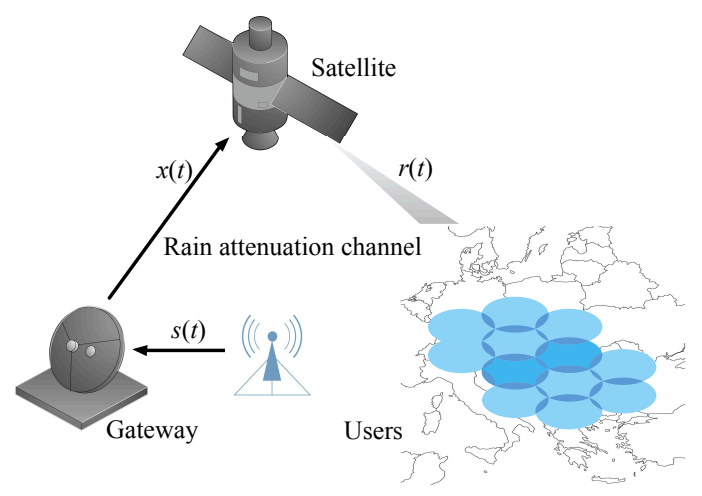

Fig. 1. System model 
where $\boldsymbol{e}_{i}=\left(e^{j \delta_{i 1}}, \ldots, e^{j \delta_{i K}}\right)^{T}$ represents the error vector. Rain-induced fading is a typical phenomenon in satellite communications. The water vapor may typically results in a high phase rotation [14]. Furthermore, as to whether the raininduced phase error is dominant or not depends on how large the deviation of the estimation error is from its prediction, which is related both to the specific choice of the estimation method and to the particular value of the delay encountered in satellite communications and similarly on how dynamically the time-varying space-ground communication channel fluctuates. Therefore, considering the rain attenuation in the air-to-ground (A2G) channel, $\hat{\boldsymbol{h}}_{i}$ can be formulated as:

$$
\hat{\boldsymbol{h}}_{i}=C \sqrt{10^{-\frac{z}{10}} \boldsymbol{b}_{i}} \odot \boldsymbol{\phi}_{i},
$$

where we have:

$$
C=\left(\frac{c}{4 \pi f d_{0}}\right)^{2} \frac{G_{r, i}}{\kappa B T} .
$$

Moreover, $f$ is the carrier frequency and $d_{0}$ represents the distance between the user and the GEO satellite, the user's receiver gain is denoted by $G_{r, i}$, while $T$ represents the receiver's noise temperature and $\kappa$ is Boltzmann's constant. Furthermore, $10^{-\frac{z}{10}}$ is the rain attenuation coefficient, where $\ln (z) \sim \mathcal{N}\left(\mu_{\text {rain }}, \sigma_{\text {rain }}^{2}\right)[15]$ and $\phi_{i}=e^{j \hat{\boldsymbol{\theta}}_{i}}$ denotes the estimated phase of the $i$-th user [15]. Additionally, $\boldsymbol{b}_{i}$ in Eq. (5) represents the beam gain, which can be approximated by [11]:

$$
b_{i k}=G_{s, k}\left(\frac{J_{1}\left(u_{k}\right)}{2 u_{k}}+36 \frac{J_{3}\left(u_{k}\right)}{u_{k}^{3}}\right)^{2},
$$

where $G_{s, k}$ is the transmitter gain of the $k$-th beam on board. Moreover, we have $u_{k}=2.07123 \frac{\sin \alpha_{k, i}}{\sin \alpha_{k, 3 \mathrm{~dB}}}$, and $\alpha_{k, i}$ is the elevation angle between the $i$-th user and the $k$-th beam center, while $\alpha_{k, 3 \mathrm{~dB}}$ denotes the half power angle.

\section{Problem Formulation}

To mitigate the cross-beam interference and to improve power efficiency, we formulate the problem as the power minimization problem under SINR constraints. However, due to the existence of phase errors, the SINR constraints are represented in the form of probability inequalities, which ensure that statistically speaking the cross-beam interference remains sufficiently low. Therefore, our robust beamforming problem can be formulated as:

$$
\begin{array}{ll} 
& \min _{\boldsymbol{w}_{i}, i=1, \ldots, I} \sum_{i=1}^{I}\left\|\boldsymbol{w}_{i}\right\|_{2}^{2} \\
\text { s.t. } & \operatorname{Pr}_{\boldsymbol{\Delta}_{i}}\left\{\operatorname{SINR}_{i} \geq \eta_{i}\right\} \geq 1-\epsilon_{i}, i=1, \ldots, I,
\end{array}
$$

where $\eta_{i}$ is the lower bound of SINR required for satisfying the QoS of the $i$-th user and $\epsilon_{i}$ is the failure probability. Hence, each user's service is fulfilled with a probability of $\left(1-\epsilon_{i}\right)$. Noting that $\left|\boldsymbol{h}_{i}^{H} \boldsymbol{w}_{i}\right|^{2}=$ $\boldsymbol{h}_{i}^{H} \boldsymbol{w}_{i} \boldsymbol{w}_{i}^{H} \boldsymbol{h}_{i}=\boldsymbol{h}_{i}^{H} \mathbf{W}_{i} \boldsymbol{h}_{i}$, the inequality $\operatorname{SINR}_{i} \geq \eta_{i}$ is equivalent to $\boldsymbol{h}_{i}^{H}\left(\frac{1}{\eta_{i}} \mathbf{W}_{i}-\sum_{j \neq i} \mathbf{W}_{j}\right) \boldsymbol{h}_{i} \geq \tau_{i}^{2}$. According to Eq. (4), the left side can be reformulated as $\boldsymbol{e}_{i}^{H}\left[\operatorname{diag}\left(\hat{\boldsymbol{h}}_{i}^{H}\right)\left(\frac{1}{\eta_{i}} \mathbf{W}_{i}-\sum_{j \neq i} \mathbf{W}_{j}\right) \operatorname{diag}\left(\hat{\boldsymbol{h}}_{i}\right)\right] \boldsymbol{e}_{i}=\boldsymbol{e}_{i}^{H} \mathbf{Z}_{i} \boldsymbol{e}_{i}$.
Hence, we achieve the first constraint in (9). After these derivations, the optimization problem can be reformulated as:

$$
\begin{array}{cl}
\min _{\mathbf{W}_{i}, i=1, \ldots, I} & \sum_{i=1}^{I} \operatorname{Tr}\left(\mathbf{W}_{i}\right) \\
\text { s.t. } & \operatorname{Pr}_{\boldsymbol{\Delta}_{i}}\left\{\boldsymbol{e}_{i}^{H} \mathbf{Z}_{i} \boldsymbol{e}_{i} \geq \tau_{i}^{2}\right\} \geq 1-\epsilon_{i}, \\
& \operatorname{Rank}\left(\mathbf{W}_{i}\right)=1, i=1, \ldots, I,
\end{array}
$$

where the positive semi-definite matrix obeys $\mathbf{W}_{i}=\boldsymbol{w}_{i} \boldsymbol{w}_{i}^{H}$, while $\mathbf{Z}_{i} \triangleq \operatorname{diag}\left(\hat{\boldsymbol{h}}_{i}^{H}\right)\left(\frac{1}{\eta_{i}} \mathbf{W}_{i}-\sum_{j \neq i} \mathbf{W}_{j}\right) \operatorname{diag}\left(\hat{\boldsymbol{h}}_{i}\right)$. Since the constraints are represented in the form of probability inequalities of complex exponential random variables, it is hard to check the associated feasibility. Therefore, we convert the complex exponential probabilistic constraints into a quadratic form relying on a linear transformation. For the quadratic form probability inequalities, Wang et al. [16] pointed out that the feasibility decreases upon increasing the SINR $\eta$. Moreover, the LTI based method rates the feasibility to be much higher than that of the probabilistic second-order cone program (SOCP) based method. Below, we will first provide a near-optimal solution of (9) relying on a relaxed chanceconstraint.

\section{Approximation And Solutions}

In this section, we first convert the problem in 97 into an SDP approximation problem via a Taylor's expansion as well as an LDI. Then, we solve the SDP relying on CVX [17] and the Gaussian randomization.

\section{A. The approximation of complex exponential Gaussian vari- ables}

First we use the quadratic Gaussian variables to approximate the complex exponential Gaussian variables via a Taylor's expansion as well as linear mapping, which is embodied in Lemma 1.

Lemma 1 For complex exponential Gaussian variables $\boldsymbol{e}=\left(e^{j \delta_{1}}, \ldots, e^{j \delta_{I}}\right), \delta_{i} \in \mathbb{R}, i=1, \ldots, I$, then $\boldsymbol{e}^{H} \mathbf{Z} \boldsymbol{e}$ can be approximately represented by its second-order Taylor's series as:

$$
\boldsymbol{e}^{H} \mathbf{Z} \boldsymbol{e} \doteq \sum_{i, j} \mathbf{Z}_{i, j}+\boldsymbol{\Delta}^{T} f(\mathbf{X}) \boldsymbol{\Delta}+\boldsymbol{\Delta}^{T} g(\mathbf{Y})
$$

where $\mathbf{X} \in \mathbb{S}^{K}$ is the real part of $\mathbf{Z}$, while $\mathbf{Y} \in \mathbb{K}^{K}$ is the imaginary part of $\mathbf{Z}$. The functions $f: \mathbb{R}^{K \times K} \rightarrow \mathbb{R}^{K \times K}$ and $g: \mathbb{R}^{K \times K} \rightarrow \mathbb{R}^{K}$ constitute a linear mapping defined by:

$$
\begin{gathered}
{[f(\mathbf{X})]_{m, n}= \begin{cases}\mathbf{X}_{m, n}-\sum_{l} \mathbf{X}_{m, l}, & m=n, \\
\mathbf{X}_{m, n}, & m \neq n,\end{cases} } \\
{[g(\mathbf{Y})]_{m}=2 \sum_{l} \mathbf{Y}_{m, l} .}
\end{gathered}
$$

The proof of Lemma 1 can be provided with reference to [10]. Relying on Lemma 1 in Eq. (10), the constraint in 9) can be 
relaxed into a probability restriction associated with quadratic Gaussian variables, in the form of:

$$
\begin{aligned}
& \operatorname{Pr}\left\{\boldsymbol{e}_{i}^{H} \mathbf{Z}_{i} \boldsymbol{e}_{i}<\tau_{i}^{2}\right\} \doteq \\
& \operatorname{Pr}\left\{\boldsymbol{\Delta}_{i}^{T} f\left(\mathbf{X}_{i}\right) \boldsymbol{\Delta}_{i}+\boldsymbol{\Delta}_{i}^{T} g\left(\mathbf{Y}_{i}\right)+\sum_{j, k} \mathbf{Z}_{i,[j, k]}-\tau_{i}^{2}<0\right\}<\epsilon_{i},
\end{aligned}
$$

where $\mathbf{Z}_{i,[j, k]}$ represents the $(j, k)$-th element of $\mathbf{Z}_{i}$. In the following, we aim for eliminating the probability constraint, which may yield a quadratic constraint based on LDI.

\section{B. The conservative approximation of probability inquality}

Lemma 2 For $\widetilde{\Delta} \sim \mathcal{N}\left(\mathbf{0}, \mathbf{I}_{K}\right)$, we have:

$$
\begin{aligned}
& \operatorname{Pr}\left\{\widetilde{\boldsymbol{\Delta}}^{T} \mathbf{Q} \widetilde{\boldsymbol{\Delta}}+\operatorname{Re}\left\{\widetilde{\boldsymbol{\Delta}}^{T} \boldsymbol{r}\right\}+s \leq 0\right\} \\
\leq & \left\{\begin{array}{l}
\exp \left(-\frac{\zeta^{2}}{4 D^{2}}\right), \quad 0<\zeta \leq 2 \lambda \nu D, \\
\exp \left(-\frac{\lambda \nu \zeta}{D}+(\lambda \nu)^{2}\right), \quad \zeta>2 \lambda \nu D,
\end{array}\right.
\end{aligned}
$$

where $\nu=\left[-(\lambda+\log (1-\lambda)) / \lambda^{2}\right]^{\frac{1}{2}}, \zeta=\operatorname{Tr}(\mathbf{Q})+s$ and $D=\nu\|\mathbf{Q}\|_{F}+\frac{1}{2 \sqrt{2}}\|\boldsymbol{r}\|_{2}$. The proof of Lemma 2 can be provided with reference to [16]. Relying on Lemma 2, let the right side of the inequality in (14) be less than $\epsilon$, which is equivalent to $2 \sqrt{-\ln \epsilon} D \leq \zeta<2 \lambda \nu D$ or $\zeta \geq \max \left\{2 \lambda \nu D,\left(\lambda \nu+\frac{-\ln \epsilon}{\lambda \nu}\right) D\right\}$. Then we have:

$$
\frac{\zeta}{D} \geq\left\{\begin{aligned}
2 \sqrt{-\ln \epsilon}, & \lambda \nu>\sqrt{-\ln \epsilon}, \\
\left(\frac{-\ln \epsilon}{\lambda \nu}+\lambda \nu\right), & \lambda \nu \leq \sqrt{-\ln \epsilon} .
\end{aligned}\right.
$$

The inequality in 15 grants us the freedom of selecting $\lambda$. Note that $\lambda \nu+\frac{-\ln \epsilon}{\lambda \nu}$ reaches the minimum value of $2 \sqrt{-\ln \epsilon}$ when $\lambda \nu=\sqrt{-\ln \epsilon}$. Therefore, to minimize the value of $\zeta / D$, which can be viewed as the least conservative approximation of the chance-constraint, let $\lambda \nu=\sqrt{-\ln \epsilon}$, yielding:

$$
\lambda+\ln (1-\lambda)=\ln \epsilon,
$$

which has one root in the range of $(\sqrt{1-\epsilon}, 1)$, that can be found by the classic bisection method, since the left side of Eq. (16) decreases monotonically when $\lambda>0$. Hence, the inequality in (15) reduces to $\zeta>2 \sqrt{-\ln \epsilon} D$. By introducing a pair of auxiliary variables $x$ and $y$, the outage constraint can be rewritten as:

$$
\begin{aligned}
s+\operatorname{Tr}(\mathbf{Q}) & \geq 2 \sqrt{-\ln \epsilon}(x+y), \\
\frac{1}{2 \sqrt{2}}\|\boldsymbol{r}\|_{2} & \leq x \\
\nu\|\mathbf{Q}\|_{F} & \leq y .
\end{aligned}
$$

Obviously we have $\Delta=\mathrm{C}^{\frac{1}{2}} \widetilde{\Delta}$. Meanwhile, in our formulation, let us introduce following definitions $s=\sum_{j, k} \mathbf{Z}_{j, k}-\tau^{2}$, $\mathbf{Q}=\mathbf{C}^{\frac{1}{2}} f(\mathbf{X}) \mathbf{C}^{\frac{1}{2}} \triangleq \mathbf{C}^{\frac{1}{2}} \mathbf{F} \mathbf{C}^{\frac{1}{2}}$ and $\boldsymbol{r}=\mathbf{C}^{\frac{1}{2}} g(\mathbf{Y}) \triangleq \mathbf{C}^{\frac{1}{2}} \mathbf{G}$. Therefore, relying on Lemma 1 and Lemma 2, the original optimization problem in 9 can be reformulated as:

$$
\begin{aligned}
& \min _{\mathbf{W}_{i}, x_{i}, y_{i}, i=1, \ldots, I} \sum_{i=1}^{I} \operatorname{Tr}\left(\mathbf{W}_{i}\right) \quad \text { s.t. } \\
& C 1: \sum_{j, k} \mathbf{Z}_{i,[j, k]}-\tau_{i}^{2}+\operatorname{Tr}\left(\mathbf{C}_{i}^{\frac{1}{2}} \mathbf{F}_{i} \mathbf{C}_{i}^{\frac{1}{2}}\right) \geq 2 \sqrt{-\ln \epsilon_{i}}\left(x_{i}+y_{i}\right), \\
& C 2: \frac{1}{2 \sqrt{2}}\left\|\mathbf{C}_{i}^{\frac{1}{2}} \mathbf{G}_{i}\right\|_{2} \leq x_{i},
\end{aligned}
$$

$C 3: v_{i}\left\|\operatorname{vec}\left(\mathbf{C}_{i}^{\frac{1}{2}} \mathbf{F}_{i} \mathbf{C}_{i}^{\frac{1}{2}}\right)\right\|_{2} \leq y_{i}$,

$C 4: \mathbf{Z}_{i}=\mathbf{X}_{i}+j \mathbf{Y}_{i}$,

$C 5: \mathbf{F}_{i}=f\left(\mathbf{X}_{i}\right), \mathbf{G}_{i}=g\left(\mathbf{Y}_{i}\right)$,

$C 6: \mathbf{W}_{i} \succeq 0$,

$C 7: \operatorname{Rank}\left(\mathbf{W}_{i}\right)=1, \quad i=1, \ldots, I$.

\section{Gaussian randomization}

Due to the rank constraints, the problem is still non-convex. Motivated by the methodology in [18], in this section, we give the Gaussian randomization algorithm for non-convex problem (18), which is give in Algorithm 1. First, by dropping the rank constraint, the optimization problem reduces to a convex SDP, which can be solved by CVX. If the rank of the solution $\hat{\mathbf{W}}_{i}$ is 1 , then the optimal $\boldsymbol{w}_{i}$ can simply be found by eigen-decomposition, i.e. 8. However, if the rank of $\hat{\mathbf{W}}_{i}$ is higher than 1 , we generate $\left(\boldsymbol{w}_{1}, \ldots, \boldsymbol{w}_{I}\right)$ by using $\hat{\mathbf{W}}_{1}, \ldots, \hat{\mathbf{W}}_{I}$ as the covariance matrix of the Gaussian distribution and scale them to satisfy the constraints, i.e. $2 \sim 5$, in each loop. Moreover, $L$ is the maximum times of Gaussian sampling.

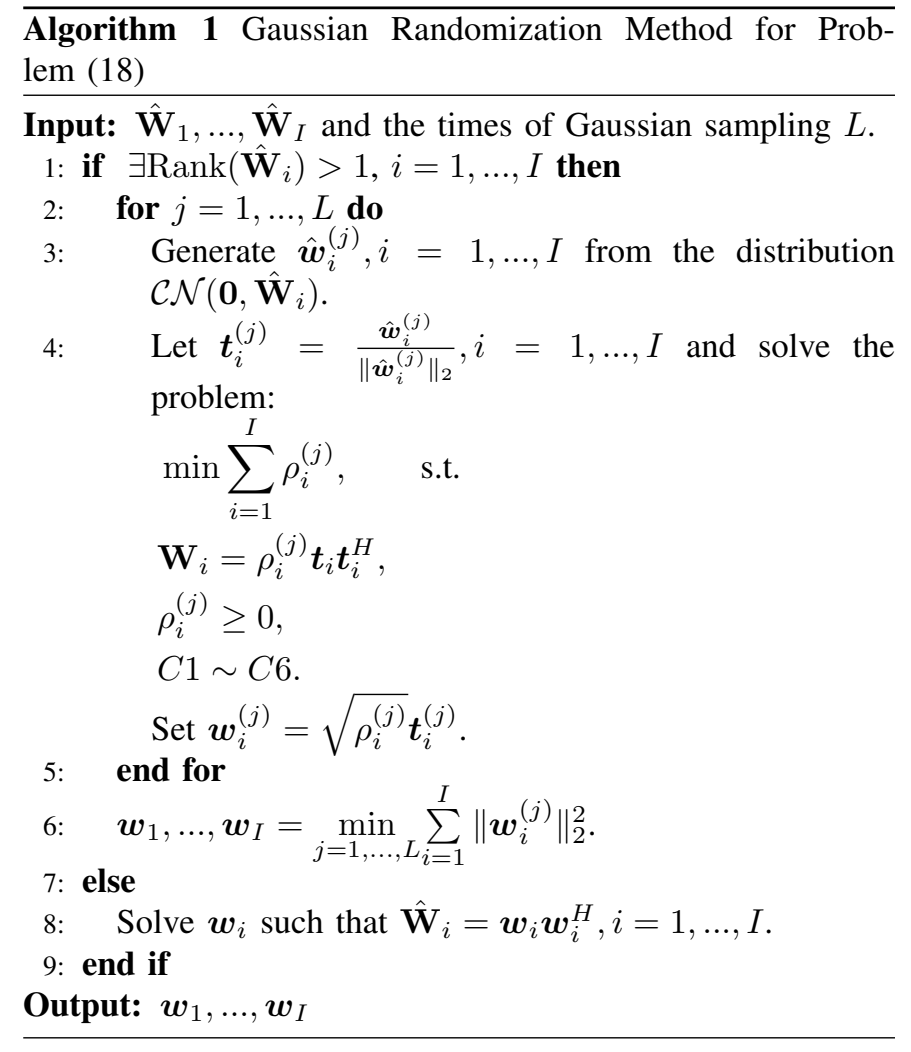

\section{Simulation Results}

In our simulations, we benchmark the performance of our proposed algorithm in comparison to both a non-robust method, as well as to the central limit theorem aided algo- 
TABLE I

PARAMETERS

\begin{tabular}{l|l}
\hline \hline Parameters & Value [11 \\
\hline \hline Orbit altitude of GEO & $d_{0}=35786(\mathrm{Km})$ \\
\hline Downlink carrier frequency & $f=20(\mathrm{GHz})$ \\
\hline Bandwidth & $B=50(\mathrm{MHz})$ \\
\hline Number of beams & $K=5$ \\
\hline Boltzmann's constant & $\kappa=1.38 \times 10^{-23}(\mathrm{~J} / \mathrm{K})$ \\
\hline Satellite transmitting gain & $G_{s, k}=35(\mathrm{dBi})$ \\
\hline User received gain per noise temperature & $G_{r, i} / T=34(\mathrm{~dB} / \mathrm{K})$ \\
\hline Half power angle & $\alpha_{3 \mathrm{~dB}}=0.4^{\circ}$ \\
\hline
\end{tabular}

rithm ${ }^{1}$ (denoted as CLT) of [11] and to the Bernstein-type inequality assisted algorithm 2 (termed as BTI) of [10].

In Table I, we summarize the essential parameters of the simulations. We set the noise power $\tau^{2}$ to 1 and the phase estimate $\phi$ to 1 . The phase perturbations obey a zero-mean Gaussian distribution with the correlation matrix of $\mathbf{C}=\sigma \mathbf{I}$, where $\sigma=5^{\circ}$. The location of $I=5$ users is generated randomly within the coverage of $K=5$ beams. In the convex optimization, we relax the rank-one constraint of 99 with the aid of Gaussian randomization. We use the CVX tool to solve our SDP subproblem. The number of Gaussian sampling is set to $L=10$.

Fig. 2 shows the minimum total power consumption required for satisfying the lower bound SINR constraint $\eta$ ranging from $0 \mathrm{~dB}$ to $5 \mathrm{~dB}$ parameterized by the outage parameter values of $\epsilon=0.01, \epsilon=0.05$ and $\epsilon=0.1$, respectively. We disregard some values of the BTI method and the CLT method, which exceed 120 because of its extreme power consumption. We can conclude from Fig. 2 that our proposed algorithm is the most energy-efficient one, outperforming both the BTI and CLT, whilst meeting the SINR requirement.

In Fig. 3, we use the classic Monte Carlo method for evaluating the actual outage probability versus the lower bound of the SINR $\eta$ ranging from $0 \mathrm{~dB}$ to $5 \mathrm{~dB}$ in conjunction with the theoretical values of $\epsilon=0.01, \epsilon=0.05$ and $\epsilon=0.1$. Moreover, the gap between the actual and the theoretical outage probability reveals, how conservative our approximation is.

The actual outage probability in the context of the nonrobust method ranges from 0.1 to 0.4 , which implies that the outage probability is sensitive to phase perturbations upon increasing the SINR. This which underlines the importance of an accurate phase estimation and a robust precoding scheme, which can compensate the SINR degradation caused by phase perturbations with the aid of some extra power consumption. The actual outage probability in the context of CLT is nearzero due to the high power consumption compared to both our proposed method and to the BTI. Furthermore, our proposed method is characterized by a low power consumption as well as by an acceptable outage probability, compared to the BTI.

\footnotetext{
${ }^{1}$ CLT approximates the distribution of quadratic form of complexexponential Gaussian random variables with a Gaussian distribution relying on the central limit theorem.

${ }^{2}$ BTI solves the beamforming problem without considering channel interference based on Taylor approximation and Bernstein-type inequalities.
}

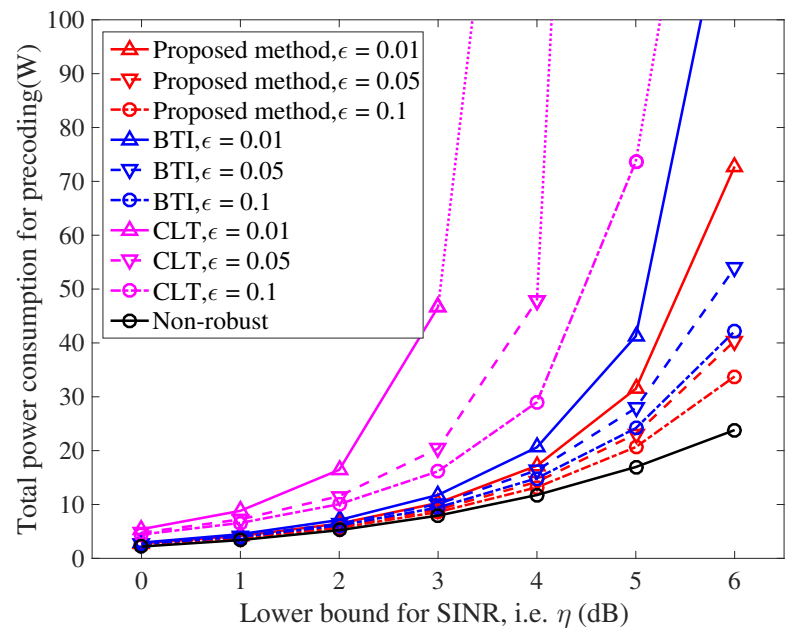

Fig. 2. Total power consumption versus lower bound of SINR $(\eta)$ parameterized by outage probability $(\epsilon)$.

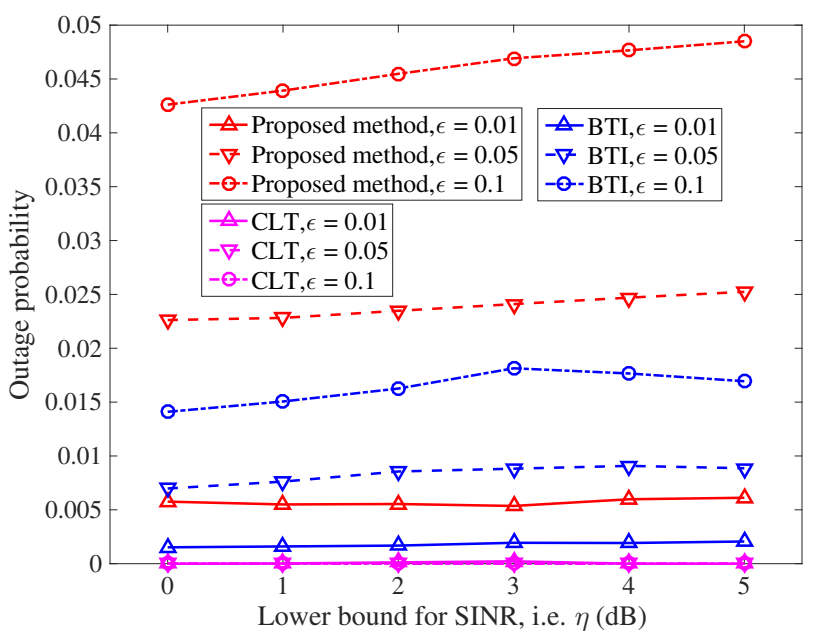

Fig. 3. Actual outage probability versus lower bound of SINR $(\eta)$ parameterized by outage probability $(\epsilon)$.

Theoretically, if we scale $\boldsymbol{w}_{i}, i=1, \ldots, I$ in Eq. (3) by a common factor of $\rho>1$, then the SINR can be deemed to be an increasing function of the parameter $\rho$. Therefore, the increased power consumption may be expected to result in a high probability of satisfying the SINR constraint. Furthermore, as for the approximation of the probability constraint, the central limit theorem requires a sufficiently large number of variables due to its asymptotic nature, which indicates that the approximation invoked for a small-scale beamforming problem may give rise to an unmeasurable approximation error. Hence, it may require excessive power to fulfill its overly conservative approximation. In contrast, we use the second-order Taylor expansion, which is an accurate approximation near the point where it expands. Moreover, small perturbations occur with a high probability since a zero-mean gaussian variable having a small deviation is typically close to zero, which implies that our approximation is accurate with a high probability. As to the approximation of the probability inequality, our proposed method depends on the large deviation inequality of Lemma 2, while the BTI method depends on the accuracy 
of the Bernstein-type inequality. Observe from the simulation results of Fig. 3 that the large deviation inequality outperforms the Bernstein-type inequality in terms of avoiding the overly conservative approximation of the probability constraint characterized by the gap between the actual and the theoretical outage probability. Finally, the computational complexities of the three algorithms are identical, because they all rely on the SDP problem having the same dimension, which can be solved within a worst-case complexity on the order of $\mathcal{O}\left[\max (K, I) K^{\frac{1}{2}} \log \left(\frac{1}{\omega}\right)\right]$, where $\omega$ represents the solution accuracy [18].

\section{Conclusions}

In this paper, we proposed robust beamforming for multibeam satellite communication in the face of phase perturbations. We formulated the robust beamforming as a minimal power consumption problem under a probability constraint. Furthermore, relying on Taylor's expansion and on LDI, we relaxed the chance-constrained problem into an SDP. Finally, our simulation results demonstrated that the proposed algorithm is beneficial in terms of both providing a constraint relaxation and yielding a high energy efficiency.

\section{REFERENCES}

[1] D. Christopoulos, S. Chatzinotas, and B. Ottersten, "Multicast multigroup precoding and user scheduling for frame-based satellite communications," IEEE Transactions on Wireless Communications, vol. 14, no. 9, pp. 4695-4707, Sep. 2015.

[2] V. Joroughi, M. Á. Vázquez, and A. I. Pérez-Neira, "Generalized multicast multibeam precoding for satellite communications," IEEE Transactions on Wireless Communications, vol. 16, no. 2, pp. 952-966, Feb. 2017.

[3] G. Zheng, S. Chatzinotas, and B. Ottersten, "Generic optimization of linear precoding in multibeam satellite systems," IEEE Transactions on Wireless Communications, vol. 11, no. 6, pp. 2308-2320, Jun. 2012.

[4] V. Joroughi, M. B. Shankar, S. Maleki, S. Chatzinotas, J. Grotz, and B. Ottersten, "Designing joint precoding and beamforming in a multiple gateway multibeam satellite system," in IEEE Wireless Communications and Networking Conference (WCNC), Barcelona, Spain, Apr. 2018, pp. $1-6$.

[5] C. Jiang, X. Zhu, L. Kuang, Y. Qian, and J. Lu, "Multimedia multicast beamforming in integrated terrestrial-satellite networks," in 13th International Wireless Communications and Mobile Computing Conference (IWCMC), Valencia, Spain, Jun. 2017, pp. 340-345.

[6] V. Bankey and P. K. Upadhyay, "Ergodic capacity of multiuser hybrid satellite-terrestrial fixed gain af relay networks with cci and outdated csi," IEEE Transactions on Vehicular Technology, DOI:10.1109/TVT.2018.2793420, 2018.

[7] M. Á. Vázquez Oliver, M. Bhavani Shankar, C. Kourogiorgas, P.-D. Arapoglou, V. Icolari, S. Chatzinotas, A. D. Panagopoulos, and A. I. Pérez Neira, "Precoding, scheduling and link adaptation in mobile interactive multibeam satellite systems," IEEE Journal on Selected Areas in Communications, vol. 36, no. 5, pp. 971-980, May 2018.

[8] J. Wang and D. P. Palomar, "Worst-case robust MIMO transmission with imperfect channel knowledge," IEEE Transactions on Signal Processing, vol. 57, no. 8, pp. 3086-3100, Aug. 2009.

[9] B. Li, Z. Fei, Z. Chu, F. Zhou, K.-K. Wong, and P. Xiao, "Robust chance-constrained secure transmission for cognitive satelliteterrestrial networks," IEEE Transactions on Vehicular Technology, DOI:10.1109/TVT.2018.2791859, 2018.

[10] M. C. Yue, S. X. Wu, and M. C. So, "A robust design for MISO physicallayer multicasting over line-of-sight channels," IEEE Signal Processing Letters, vol. 23, no. 7, pp. 939-943, Jul. 2015.

[11] A. Gharanjik, S. M. R. Bhavani, P. D. Arapoglou, M. Bengtsson, and B. Ottersten, "Robust precoding design for multibeam downlink satellite channel with phase uncertainty," in IEEE International Conference on Acoustics, Speech and Signal Processing, South Brisbane, Australia, Apr. 2015, pp. 3083-3087.
[12] A. El-Keyi and B. Champagne, "Collaborative uplink transmit beamforming with robustness against channel estimation errors," IEEE Transactions on Vehicular Technology, vol. 58, no. 1, pp. 126-139, 2009.

[13] E. Casini, R. D. Gaudenzi, and A. Ginesi, "DVB-S2 modem algorithms design and performance over typical satellite channels," International Journal of Satellite Communications and Networking, vol. 22, no. 3, pp. 281-318, Mar. 2004.

[14] E. Matricciani, "A relationship between phase delay and attenuation due to rain and its applications to satellite and deep-space tracking," IEEE Transactions on Antennas and Propagation, vol. 57, no. 11, pp. 36023611, Nov. 2009.

[15] J. Arnau, D. Christopoulos, S. Chatzinotas, C. Mosquera, and B. Ottersten, "Performance of the multibeam satellite return link with correlated rain attenuation," IEEE Transactions on Wireless Communications, vol. 13, no. 11, pp. 6286-6299, Nov. 2014.

[16] K. Y. Wang, M. C. So, T. H. Chang, W. K. Ma, and C. Y. Chi, "Outage constrained robust transmit optimization for multiuser MISO downlinks: Tractable approximations by conic optimization," IEEE Transactions on Signal Processing, vol. 62, no. 21, pp. 5690-5705, Nov. 2014.

[17] M. Grant, S. Boyd, and Y. Ye, "CVX: Matlab software for disciplined convex programming," 2008.

[18] Z. Q. Luo, W. K. Ma, M. C. So, Y. Ye, and S. Zhang, "Semidefinite relaxation of quadratic optimization problems," IEEE Signal Processing Magazine, vol. 27, no. 3, pp. 20-34, Mar. 2010. 